ISSN: 2386-3919 - e-ISSN: 2386-3927

DOI: https://doi.org/10.14201/et2017352127139

\title{
CAPITAL CULTURAL Y AVENTURA PERSONAL EN LA CONSTRUCCIÓN DE LA IDENTIDAD DE GÉNERO
}

\author{
Cultural capital and personal adventure \\ in the construction of gender identity
}

Carlos Rosales López

Correo-e: carlos.rosales@usc.es

Recibido: 21-5-2017; Aceptado: 30-7-2017; Publicado: 30-11-2017

Ref. Bibl. CARLOS ROSALES LÓPEZ. Capital cultural y aventura personal en la construcción de la identidad de género. Enseñanza \& Teaching, 35, 2-2017, 127-139.

RESUMEN: En este trabajo se pone de relieve cómo la construcción de la identidad de género constituye una tarea personal que se desarrolla a lo largo de la vida y en los diversos escenarios en que ésta tiene lugar. La influencia de la herencia cultural tradicional en dichos escenarios (familia, escuela, comunidad...) es intensa y se manifiesta de forma ambiental, así como a través de intervenciones intencionales directas sobre las personas. Dicha influencia se polariza entre los extremos de lo masculino y lo femenino con un matiz claramente diferenciador y discriminador en el caso de lo femenino. Al tiempo, se olvidan o menosprecian los sentimientos, las dificultades y aspiraciones de cada persona, así como sus trayectorias de vida.

Para la realización de este trabajo se reflexiona sobre recientes investigaciones en el ámbito de la igualdad en relación con la tradicional línea de investigación sobre influencia del capital cultural en la conformación de la personalidad. En sus conclusiones se considera que innovar en la construcción del rol de género implica romper con tales presiones limitantes y apostar por el progreso hacia la libertad y la igualdad entre los seres humanos.

Palabras clave: identidad de género; cultura social; familia; escuela; orientación laboral; iniciativa personal. 
SUMMARY: This paper highlights how the construction of gender identity is a personal task that develops throughout life and in various scenarios in whit it takes place. The influence of traditional cultural heritage in these scenarios (family, school, community...) is intense and manifest environmental way and through direct intentional interventions. The influence is polarized between the ends of the male female with a cleary differentiating and discriminatory toward the feminine genre. They forget or underestimate the feeling and difficulties of each person and their life trajectories.

For the realization of this work we reflect on recent research in the field of equality in relation to the traditional line of research on the influence of cultural capital on the formation of personality. The paper concludes by considering that innovation in the construction of gender role involves breaking with such limiting pressure and bet on the progress toward freedom and equality between human beings.

Key words: gender identity; social culture; family; school; work orientation; personal initiative.

\section{PAPEL ACTIVO DE LA PERSONA}

La construcción de la identidad de género se ha considerado tradicionalmente como resultado de una influencia unidireccional predominante de los contextos de vida sobre la conformación de la personalidad. No obstante, en estos momentos es preciso señalar que la investigación sugiere una importante alternativa a tal reducido concepto. La persona no se limita a desempeñar un papel pasivo, receptor de influencias, sino que desde el comienzo de su vida interactúa de manera directa con su entorno vital, constituyéndose en un agente activo en la construcción de su propia identidad, que no permanece de manera estable, sino que evoluciona con el tiempo, atravesando diversas etapas madurativas.

La influencia de los contextos de vida es muy variada. El nivel cultural y socioeconómico a que pertenece la persona la hace heredera de diferentes posibilidades y orientaciones en la conformación de su identidad. Dicha herencia se va a transmitir de muy diversas maneras, desde la constante presencia ambiental en entornos de vida, con un carácter por lo tanto indirecto, con un efecto de "ósmosis", hasta muy variadas formas de intervención directa de los miembros de dichos entornos (familiares, docentes, políticos, informadores de los MCs...).

Dado el carácter plural de la sociedad en estos momentos, es frecuente la confluencia, interacción y confrontación de orientaciones entre familia y colegio, entre éste y los Mcs, entre culturas diversas en la comunidad...

Dada la naturaleza de las influencias, a veces en interferencia, que la persona va a experimentar en función de esta compleja herencia cultural, la conformación de su identidad de género se puede vivir por el niño y el joven como una difícil aventura no desprovista de importantes riesgos personales. Deberá tratar de armonizar herencia cultural e influencia social con sus propias ideas y proyectos. Imaginemos, en este sentido, las dificultades de personas con una clara tendencia 
homosexual en un ambiente fuertemente polarizado en la heterosexualidad. $O$ la vivencia de identidad de género por la mujer que aspira a la igualdad, en entornos laborales fuertemente discriminadores. Imaginemos la situación conflictual de jóvenes en contextos interculturales con tendencias dispares (cristianos, musulmanes...).

Parece necesario realizar algún tipo de aclaración conceptual en torno a la identidad y el rol de género. Se podría considerar, como indican diversos autores (Rouyer, 2014), que la identidad constituye la experiencia privada del rol de género y que éste está constituido por todo aquello que la persona dice o hace para indicar el grado en que es hombre o mujer. La identidad de género, como estado subjetivo, se compone de una compleja serie de creencias y sentimientos sobre su masculinidad o feminidad. En estos momentos se considera que dicha identidad no deriva solamente de factores innatos o de estímulos del entorno, sino de un complejo proceso en el que interactúan componentes biológicos, sociales y psicológicos. Hasta el momento, se ha concedido poco interés a los factores afectivos. La naturaleza de la identidad de género está estrechamente vinculada al rol correspondiente, es decir, a las conductas que la persona desempeña como manifestación de su identidad.

En la conformación de la identidad de género existe, pues, un componente activo, interactivo de la persona con su entorno, y se desarrolla, desde una perspectiva temporal, a lo largo de toda su vida, dándose un proceso de adaptación que, en términos de la teoría evolutiva de J. Piaget, podríamos considerar constituido por la doble actividad de asimilación y acomodación. En definitiva, la persona constituye un agente activo en la construcción de su propia identidad. Y hay que considerar que, en la actualidad, las tradicionales identidades y roles, entre los polos de lo masculino y lo femenino, se encuentran considerablemente diversificadas en una variedad de conceptos.

Según Rouyer (2014), la naturaleza de las interacciones que el niño mantiene con sus padres entre los dieciocho y los veinticuatro meses contribuye al desarrollo de su identidad sexual. Entre los dos y los tres años será capaz de identificar a las personas de sexo masculino y femenino y su nivel de conocimiento y discriminación se extiende progresivamente en ámbitos como los juguetes, vestidos, objetos y actividades, de modo que entre los seis y los siete años es comparable al de los adultos.

Su adscripción a una identidad y rol de género no tiene carácter estable. Piensa que se puede cambiar de grupo en función de características, sobre todo socioculturales, manifestando flexibilidad respecto a la pertenencia a uno u otro (Dafflou y Novelle, 2010). En las explicaciones que proporcionan sobre las diferencias de género, los niños de cinco a doce años utilizan más argumentos psicológicos que biológicos o sociales (Tostain, 2010). La adhesión a un género a esta edad está en relación y constituye un predictor de la que manifestará años después. Así, según Granie (2010), los niños que a los tres años manifiestan una clara adhesión a un género van a manifestar diez años después un alto nivel de pertenencia al grupo correspondiente. 
En el estudio de la construcción de la identidad de género, se considera en la actualidad que es necesario superar la tradicional dicotomía masculino/femenino, para profundizar en el conocimiento de variaciones interindividuales e intraindividuales, contemplando una trayectoria de identidad, en este último caso, a lo largo de la vida (Prêteur, 2010). Se estima relevante, asimismo, tomar en consideración el punto de vista de la persona, conocer cómo niños y jóvenes interpretan los componentes biológicos, sociales y psicológicos; cómo viven las diferentes experiencias de socialización en relación a múltiples características (cultural, étnica, generacional, etc.).

\section{INFLUENCIA FAMILIAR}

Desde los primeros momentos de la vida, el entorno familiar ejerce una múltiple influencia sobre la persona, induciendo en ella un determinado rol: masculino o femenino. Los vestidos, los juguetes, la interacción que se mantiene con el niño o la niña presentan marcadas diferencias. Vestidos en rosa y azul, juegos de muñecas y automóviles, una interacción en la que se inducen modelos de relación y sentimientos (una niña dulce, un niño fortachón...). Los primeros aprendizajes en todos los ámbitos de la vida aparecen marcados en el hogar por la influencia del ambiente y por las intervenciones directas, diferenciadoras, que se mantienen con el niño y la niña. Está bien visto que la niña realice tareas en el hogar igual que su madre, así como que el niño acompañe y ayude en sus tareas al padre. Los/ as niños/as llegan a considerar normal que sus madres, después de trabajar fuera de casa, realicen las tareas del hogar, pero no lo ven tan lógico en el caso de los padres. Se considera normal que las madres cuiden de las personas enfermas en el hogar, pero es más raro que se asocie con naturalidad al padre.

El ambiente, en general discriminador, que se vive en el hogar se manifiesta claramente en actividades cotidianas específicas. En un estudio realizado por Crowley (2001), se pone de relieve una atención muy diferente de los padres a sus hijos entre uno y ocho años. A los niños les proporcionan más explicaciones que a las niñas y, además, dichas explicaciones son más detalladas, ricas y variadas. En cuanto a las conductas de riesgo, las chicas reciben más atención y apoyo, mientras que a los chicos se les anima a arriesgarse y actuar sin ayuda.

La influencia del hogar en la constitución de la identidad y del rol de género puede variar cualitativamente en función del tipo de familia en que se desarrolla el niño/a. La familia convencional, constituida por padre, madre, hermanos, abuelos... en estos momentos se diversifica considerablemente. Es frecuente que la familia esté constituida solamente por los padres y un hijo o dos. Es creciente, en estos momentos también, el número de familias monoparentales por diversas causas como la separación. Y pueden darse también familias constituidas por parejas del mismo sexo. Evidentemente, en todos estos casos, la influencia va a ser cualitativamente diferente y se precisa investigar sobre las diversas situaciones y su influencia en la persona. 
La influencia de la cultura familiar se va a ejercer de forma predominante a través de la comunicación, de los modos de vida, del control que se tenga en el hogar del uso de los medios de comunicación... En todos estos ámbitos se trasladarán mensajes, orientaciones, directrices respecto a la conformación de la identidad de género. Pero la actuación familiar va a desbordar ampliamente el escenario del hogar para proyectarse también en el de la escuela y la comunidad. Así, los padres van a intervenir en la elección del colegio al que acudirán sus hijos/as y, a veces, la variable de educación en género desempeña un importante papel en dicha elección. Una vez escolarizados sus hijos/as, el seguimiento que los padres realizan de la evolución de su formación, a través de la relación con los tutores correspondientes, va a constituir otra característica de interés. La realización de los deberes, directamente con sus hijos o a través de otras personas, cuando no tienen tiempo o no están capacitados, constituye otra forma y ocasión de influencia. Respecto a la comunidad social, podríamos hacer un análisis similar. Los padres pueden controlar, hasta cierto punto, el tipo de actividades que sus hijos/as realizan en el contexto de la comunidad en horario extraescolar. A nivel medio y superior, la situación socioeconómica de los familiares va a hacer posible en mayor o menor medida los viajes y estancias de sus hijos/as en el extranjero o en otras comunidades... Y estas posibilidades a nivel cultural tienen una indudable proyección en la constitución del rol de género de sus hijos.

\section{ENTRE LA FAMILIA Y LA ESCUELA}

Así como en los primeros momentos de la vida la familia ocupa un lugar estelar en la influencia sobre el niño/a, a medida que éste crece, van siendo otros los escenarios que pasan a influir también en su formación y, por lo tanto, en su identidad y rol de género. Tradicionalmente se han identificado como grandes escenarios de vida y educación la familia, la escuela y la comunidad, con la diversidad de instituciones que la conforman. Y en algunos países tendemos a calificar de escuelas a las instituciones de acogida de los niños/as desde los primeros momentos de vida, como alternativas a la familia. Estas instituciones de acogida (escuelas infantiles, escuelas maternales, jardines de infancia, guarderías...) presentan unas características puente entre el hogar y la escuela. En realidad, se aproximan más a las características de un hogar, en el que el niño/a convive con otros niños/as de su edad.

Una característica de notable interés en estas instituciones de acogida lo es la importancia que en ellas tiene el rol femenino en cuanto al cuidado de los niños/ as, un indicador de continuidad con el hogar. Así, en estas instituciones se da la presencia profesional predominante de la mujer y una casi total ausencia de hombres, así como una fuerte asociación de las tareas profesionales a rasgos de cuidado materno; de ahí que los hombres que trabajan en ellas estén a veces "bajo sospecha» de que puedan cumplir con eficacia tales tareas.

Esta fuerte vinculación materna se pone de relieve, asimismo, en la relación más intensa de las profesionales con las madres de los niños/as que con sus padres, 
al punto de que, en este último caso, dicha relación suele entenderse como la mediación de un mensaje que finalmente se dirige a la madre.

No parecen registrarse, en principio, especiales preocupaciones por el desarrollo de la identidad de género en estas edades. La preocupación profesional se proyecta más en el desarrollo evolutivo del niño/a según su edad, en la adquisición de hábitos, actitudes y conocimientos en relación a sí mismo y a su entorno, a la capacidad de comunicación. Pero un análisis más profundo de la intervención de las/os profesionales con los niños/as puede revelar matices diferenciadores de importante valor cualitativo desde los primeros momentos. Se registran referencias a las niñas en aspectos como su sonrisa o su dulzura y a los niños sobre su actividad motora. En las chicas se da importancia a sus conductas y apariencia física, se las permite una mayor manifestación emocional, mientras que en los chicos se promueve su autocontrol en este sentido. La utilización de juguetes en estas instituciones es también diferenciadora. Por ejemplo, el «rincón de las muñecas» constituye un ámbito propio de las niñas, al que poco o nada se asoman los niños. Éstos, cuando lo hacen, no juegan siempre simulando tareas de cuidado, sino que a veces utilizan las muñecas como objetos, con otras finalidades o funciones lúdicas.

\section{LA ESCUELA: EDUCACIÓN PARA LA IGUALDAD. RASGOS DIFERENCIADORES}

El periodo de educación básica obligatoria, generalmente entre los seis y los doce años en países en desarrollo y hasta los dieciséis o más en otros más desarrollados, constituye la etapa del sistema educativo en la que se suele incidir con mayor intensidad en la formación de la persona en valores como la igualdad de género. No obstante, es preciso señalar la existencia de grandes desequilibrios entre zonas geográficas y países. A partir de recomendaciones de carácter universal como las realizadas por la ONU, UNESCO, UE y otros organismos internacionales, cada país miembro de los mismos contempla con distinta intensidad normas y medidas de intervención en este sentido. Pero la realidad da a conocer que en algunos países todavía existen niños y niñas sin escolarizar; que, en otros, el número de años que acuden a las escuelas es notablemente insuficiente como para adquirir una serie de aprendizajes básicos; que en aquellos en los que sí están escolarizados pueden darse grandes diferencias discriminatorias entre niños y niñas, al asistir éstas menos a la escuela por motivos de seguridad o de trabajo, etc.

En países más desarrollados, especialmente en los que se fomenta la libertad de los padres para elegir centro en el que eduquen a sus hijos/as, se produce a veces una elección de aquellos en los que se separa por género, en función de teorías como la feminista por la diferencia, que consideran que la mujer puede ser objeto de una mejor educación en contextos diferenciados.

En nuestro país, como en los de nuestro entorno en general, ha predominado la opción por una enseñanza que en principio comenzó por ser mixta con la LGE (1970) y paulatinamente se ha convertido en coeducadora, a través de la LOGSE 
(1990) y la LOE (2006), asistiéndose en estos momentos a cierto resurgimiento de tendencias diferenciadoras, al permitir la actual ley, LOMCE (2013), la subvención a centros que segregan por género.

Desde una perspectiva cualitativa, se han dado importantes pasos hacia el desarrollo de una auténtica coeducación (Subirats, 2014). Se ha progresado en el terreno de la legislación y normativa educativa, en el de la metodología y los recursos. No obstante, la investigación sobre igualdad real en las aulas y los colegios sigue siendo reticente al darnos a conocer que tanto a nivel formal como informal se producen efectos discriminadores más allá de la intención y declaración de igualdad de los profesionales de la enseñanza. Ocurre como si la enorme herencia social tradicional de diferenciación por género se introdujera a través de los resquicios que presenta la aplicación de normativas oficiales. Los profesionales de la enseñanza presentan sus propios rasgos y actitudes, que de alguna manera transmiten en función de un currículum oculto a través de la interacción que mantienen con sus alumnos/as y que se refleja en expresiones lingüísticas, en el tratamiento de temas con mayor o menor intensidad según interactúen con alumnos o alumnas, en distintas formas de proyectar la educación afectiva y emocional de unos y otras, en la imagen que se proyecta del hombre y la mujer adultos... También en las orientaciones de futuro, a veces cargadas de un sesgo sexista (tales estudios u ocupaciones más propias para la mujer o para el hombre).

La discriminación en el centro escolar puede manifestarse, asimismo, en la distribución de responsabilidades (un colegio con mayoría de profesoras en el que los cargos de responsabilidad están ocupados por profesores) y hasta en la utilización de espacios y recursos (diferencias en el acceso a zonas y medios, en las instalaciones deportivas y patios). Se puede hacer mención, así, a la existencia de un importante currículum oculto, paralelo al oficial, en la educación básica. Todo lo cual incrementa la necesidad de seguir trabajando por la implantación de una auténtica igualdad en la educación según género.

Desde una perspectiva social y cultural, las escuelas en estos momentos son instituciones predominantemente inclusivas. En ellas la herencia cultural familiar de cada alumno va a tomar contacto con las herencias culturales de sus compañeros, a veces muy diferentes. Se va a producir un enriquecimiento, diversificación e incluso interferencia de influencias en los planos formales e informales. Quizás las relaciones informales, por la creciente cantidad de tiempo que los niños/as pasan en la escuela, van a constituir un escenario de creciente protagonismo en la constitución de su rol de género. El niño/a, chico/a, va a desarrollar relaciones de compañerismo y amistad que pueden marcar significativamente su personalidad y, en definitiva, su identidad de género.

\subsection{La identidad de género y los textos escolares}

Los textos escolares constituyen el instrumento didáctico más intensamente utilizado en la etapa de educación obligatoria, hasta el punto de convertirse a veces 
en guía y fuente de las actividades a realizar por alumnos y profesores. A través de ellos se transmite una determinada imagen de la sociedad, de la cultura y de los roles de género. De aquí la necesidad de analizar sus características y la influencia que ejercen en los alumnos.

Biemmi (2015) ha realizado estudios de carácter cuantitativo y cualitativo sobre libros de texto utilizados en la actualidad en Italia y llega a conclusiones como las siguientes, fácilmente aplicables a nuestro entorno:

1. En el mundo que presentan los textos, el género masculino está mucho más representado.

2. Se dan sustanciales diferencias en los roles y características atribuidos a hombres y mujeres.

Los hombres desempeñan una amplia variedad de profesiones y son libres para moverse en los espacios públicos. Las mujeres tienen menos oportunidades de empleo y gastan la mayor parte de su tiempo en el hogar, vinculadas al rol de madre. Los hombres se describen como valientes, determinados, orgullosos y satisfechos, mientras que las mujeres aparecen como cuidadosas, dulces, pacientes y sensitivas. Las imágenes, por otra parte, confirman la desigualdad entre géneros. En general se favorece al masculino, mientras el femenino permanece en la sombra, como menos importante e interesante.

En el análisis cualitativo de las características atribuidas al hombre y a la mujer, Biemmi (2015) constata que, en el caso de la mujer, se concede mucha importancia a la belleza. Se la considera una característica femenina necesaria y fundamental según una dicotomía belleza/fealdad, con connotaciones específicas: belleza es el polo positivo y fealdad el negativo. Sin embargo, en el género masculino no se valora la belleza. Al hombre se lo valora por sus cualidades intelectuales, por lo que se produce una considerable asimetría entre hombres y mujeres. Biemmi (2015) ha podido constatar que, en los textos analizados, las características de la joven se asocian a las de la mujer adulta, así como las del joven al hombre. En el primer caso, los aspectos similares son muchos: ser buena y portarse bien, amorosa, cariñosa, bella... La joven considera a la mujer adulta como modelo e intenta imitarla. Se pone de relieve un "entrenamiento» de las jóvenes para hacerse buenas mujeres y madres.

Respecto a los estereotipos de género masculino, se pueden distinguir también estereotipos aplicables al hombre y al chico y compararlos. Al joven se lo representa como ingenuo, autónomo, activo, tenaz, independiente, valeroso, seguro de sí mismo, práctico, rudo, dinámico... Sin embargo, los rasgos que se asocian al hombre son escasos: fuerte, emprendedor, dinámico. Mientras que a la mujer se la juzga predominantemente por su belleza, al género masculino se lo caracteriza por su cultura.

En su estudio de roles, Biemmi (2015) constata que existe una mayor rigidez de estereotipos en el caso del hombre que en el de la mujer. Ésta es capaz de «escaparse» del modelo impuesto y manifestar conductas semejantes a las del hombre, mientras que éste está más rígidamente encasillado y es difícil que manifieste 
conductas de un rol socialmente femenino. Sin embargo, desde una perspectiva de desarrollo de la igualdad de género, es deseable que se camine hacia tareas compartidas, de modo que en el hogar participen de las actividades propias tanto el hombre como la mujer, así como que en otros ámbitos, predominantemente masculinos, se produzca una mayor presencia, no solo cuantitativa, sino también de responsabilidad, de la mujer.

Los textos escolares, como los libros de narrativa infantil y juvenil y los medios de comunicación social en general, deberán dar importantes pasos adelante en todos estos aspectos, si quieren contribuir a una igualdad real de género.

\section{LA ELECCIÓN DE CARRERA COMO INFLUENCIA DEL ROL SOCIAL DE GÉNERO}

La educación secundaria constituye una etapa clave en la orientación profesional de chicos y chicas, que han de elegir itinerarios de estudios de carácter profesional o bien encaminarse a la realización de carreras universitarias de distinta naturaleza. Sus elecciones pueden relacionarse, en parte, con sus expedientes académicos, con sus propias motivaciones y capacidades. Pero es indudable que éste no es el único ni el más importante de los factores que influyen en ellas. Resulta significativo al respecto que las carreras de carácter científico, técnico, industrial sean elegidas prioritariamente por los chicos, mientras que las chicas se decantan por las de carácter social, docente... (Aguirre, 2015). Se constata, en este sentido, la influencia en ellos/as de su proyecto de vida, dentro del que se incluye su identidad de género. Así, autores como Dura Bellat (1995, 2004) han podido constatar que la mayoría de chicas y muy pocos chicos se interesan en secundaria sobre cómo conciliar en el futuro su trabajo y su vida familiar. Y, en este sentido, si chicas y chicos se orientan hacia estudios y profesiones diferentes es porque se imaginan también de forma diferente la futura conciliación entre trabajo y vida familiar. Esta necesidad de conciliación inclinará a la chica a renunciar a profesiones más valoradas socialmente, porque las consideran incompatibles con las obligaciones de la vida familiar.

La investigación cualitativa, a través del seguimiento de trayectorias de vida, pone de relieve la existencia de una importante conexión entre socialización en género dentro del hogar y la proyección personal-profesional de las jóvenes en su futuro. Es en el hogar donde la joven vive y asume el rol de la mujer doblemente comprometida con las tareas de cuidado de las personas y el cumplimiento de sus obligaciones profesionales. Y es en los hogares de menor nivel socioeconómico donde más intensamente se vive esta realidad, que se refleja, asimismo, en las elecciones de las chicas pertenecientes a los mismos. Esta influencia de rol familiar, sin embargo, es menos intensa en el caso de chicas pertenecientes a un nivel socioeconómico más elevado, como ha puesto de relieve M. Court (2013) a través de su investigación con utilización de entrevistas.

Una profesión especialmente elegida por las mujeres es la docente. Se da una notable predominancia de la mujer en los niveles de educación infantil y primaria, 
una práctica equiparación con hombres en secundaria y un predominio de éstos en la superior. Se puede interpretar este hecho por la proximidad de roles profesional y materno en los niveles inferiores y por las condiciones de trabajo, probablemente más flexibles que en otras profesiones, lo que haría más viable la conciliación familiar. No obstante, como ha podido constatar M. Mareau (2014), un análisis profundo de las condiciones de trabajo pone de relieve que cuando éstas no son tan facilitadoras, sino que se atienen a normas más rígidas, como ocurre en Gran Bretaña, la mujer deja de elegir esta carrera y con frecuencia llega a abandonar el trabajo en ella, para poder atender sus responsabilidades familiares. M. Mareau (2014) destaca la existencia de diferencias entre Gran Bretaña y Francia. En los dos países los docentes sin hijos presentan itinerarios relativamente similares. Pero cuando se convierten en padres y madres, las carreras se diferencian sensiblemente, con interrupciones de recorrido y una utilización de la dedicación parcial más frecuente en las mujeres que en los hombres.

\section{IGUALDAD DE GÉNERO EN LOS MEDIOS DE COMUNICACIÓN SOCIAL}

Los medios de comunicación social constituyen un componente omnipresente en los diversos escenarios de la vida de las personas. Se utilizan con asiduidad e influyen de manera importante a través de estrategias de sensibilización, de información, de entretenimiento... De hecho, constituyen un poderoso vehículo de transferencia a las personas de la herencia cultural de nuestras comunidades. Su influencia sobre los más jóvenes es especialmente intensa si tenemos en cuenta el elevado número de horas que los utilizan y su escasa capacidad de recepción crítica y control sobre los mensajes que se transmiten. En términos generales y respecto a las características de los roles de género, se acepta comúnmente que los medios de comunicación social presentan una baja representación de la mujer respecto al hombre y que dicha representación responde a estereotipos tradicionales tanto en uno como en otro caso: mujer como madre y ama de casa, como profesional de bajo estatus; hombre como protagonista de la vida pública y de profesiones de alta estima social.

Los diversos intentos que se vienen realizando para reconducir una influencia tan intensa y discriminadora para la mujer chocan constantemente con los postulados de una sociedad neoliberal, en la que los beneficios económicos y la supuesta y tan esgrimida "libertad de expresión» se oponen a intentos gubernamentales para regular los contenidos de los programas y a las iniciativas civiles en este mismo sentido. Los Consejos Audiovisuales y otros organismos han logrado que se den pasos hacia un cierto respeto por la transmisión de valores sociales, como la igualdad de género, en franjas horarias de mayor audiencia infantil y juvenil. Todo ello, a través de convenios que tienen un marcado carácter voluntarista. Pero, en conjunto, la programación de los distintos medios y los mensajes publicitarios que la acompañan continúan teniendo un marcado carácter discriminador y sexista. 
Entre las iniciativas más destacadas para el cambio en este sentido, se puede destacar la Plataforma de Acción, surgida de la IV Conferencia Mundial sobre la Mujer (Beijing, 1995). Entre las áreas de intervención de dicha Plataforma, se encontraba la correspondiente a «La Mujer y los Medios de Comunicación». Y entre las iniciativas surgidas a raíz de su creación, se destaca la Red Internacional de Mujeres Periodistas con visión de Género.

Los Consejos Audiovisuales, que tienen su origen en los años noventa a partir del informe europeo "Televisión sin fronteras", han desempeñado asimismo un interesante papel regulador y estimulador. En nuestro país se destaca la labor del primero, creado en Cataluña (Camps, 2009). A él se debe la realización de un estudio cuantitativo sobre la presencia de la mujer en las cadenas catalanas de televisión, en 2008. Dicho estudio da a conocer una presencia de la mujer del 27,5\% frente a un $72,5 \%$ del hombre. Asimismo, el periodo de tiempo de palabra de la mujer era de 28 segundos frente a 44 del hombre. Se constata, pues, una ausencia notable de la mujer en informativos, al no alcanzar el 30\%.

Desde una perspectiva cualitativa, se pone de relieve un marcado androcentrismo de los programas, dado que es el hombre el que acapara el protagonismo de la información con carácter universal, para todos, mientras que la información que proporciona la mujer se suele limitar a temas más propios de su género. Los temas de información presentan un carácter marcadamente diferenciado. Así, la política y la economía se asocian al hombre, mientras que a la mujer se vinculan los de enseñanza, salud y temas sociales.

En conjunto, los medios de comunicación pretenden reflejar la realidad social. Y en ésta es evidente la existencia de discriminación hacia la mujer en múltiples sentidos (mayores cargas de responsabilidad en el hogar, peores condiciones de acceso a cargos profesionales de responsabilidad, desigualdad de salarios ante trabajos similares...). Ahora bien, los medios de comunicación deberían también comprometerse con dinámicas de progreso en el ámbito de los derechos humanos y más concretamente en el de la igualdad de género, subrayando los objetivos sociales, las normativas legales y los progresos que indudablemente se vienen produciendo respecto a prototipos convencionales discriminadores.

\section{CONCLUSIÓN: INNOVAR EN LA CONSTRUCCIÓN DEL ROL DE GÉNERO, ¿ES ROMPER CON LA HERENCIA RECIBIDA?}

El análisis realizado del proceso de construcción de identidad de género pone de relieve la existencia de una fuerte presión sobre la persona, desde los primeros momentos de su vida y en los diversos contextos en los que se desarrolla ésta. Dicha presión se ejerce a través de ambientes ya creados y de actuaciones directas sobre la persona. Y en estos momentos, en términos generales, parece predominar una polarización entre géneros masculino y femenino con marcadas diferencias de rol que se manifiestan tanto en el hogar como en la vida comunitaria. 
En todos estos escenarios, se pone de relieve una fuerte tendencia a influir sobre la persona para que se adhiera a uno u otro rol. Se relegan así características tan importantes como sus propios sentimientos y el hecho de que dicho rol puede experimentar significativas variaciones, tanto interindividuales como intraindividuales a lo largo de las distintas etapas de la vida, así como adquirir una naturaleza no necesariamente polarizada entre los extremos de lo masculino y lo femenino. Las formas de hacer, pensar y sentir tradicionalmente admitidas actúan como una fuerte oposición, a veces, frente a las perspectivas individuales. Y dichas formas tradicionales de considerar los roles de género son causa también de la discriminación existente entre uno y otro (tareas en el hogar, acceso a puestos de responsabilidad, remuneración y consideración social diferentes...).

Por lo general, la legislación educativa, laboral y social contiene normas avanzadas en cuanto a la libertad e igualdad de género. Pero estas normas con frecuencia carecen de la proyección real que cabría esperar.

La institución escolar, orientada generalmente por dichas normas renovadoras en lo educativo, constituye en estos momentos una institución avanzada, pero aun en ella, usos y costumbres tradicionales y recursos como los libros de texto actúan a modo de currículo oculto frente al progreso. La escuela, sin embargo, no puede renunciar a formar a sus alumnos/as en la autonomía, la capacidad de iniciativa, la libertad de pensamiento y sentimiento y la igualdad.

Llegamos así a la conclusión de que innovar, mejorar el proceso de construcción del rol de género, constituye un proceso aún hoy revolucionario, en el que la reconsideración crítica de fuertes influencias culturales del pasado constituye un componente fundamental.

\section{REFERENCIAS BIBLIOGRÁFICAS}

Aguirre, A. (2015). Claves para educar en igualdad. Revista Qurriculum, 8, 75-89.

Biemmi, I. (2015). The imagery of gender in Italian textbooks. Research into primary school books. Foro de Educación, 13 (18), 15-35.

Camps, V. (2009). La educación en medios, más allá de la escuela. Comunicar, 32, XVI, 140-145.

Conseil de l'Audiovisual de Catalunya: Informe sectorial trimestral: La presencia de les dones en la información: octubre-decembre, 2008, marzo, 2009.

Court, M.; Bertrand, J.; Bois, G.; Henri-Panabiere, G. y Vanhee, O. (2013). L'orientation scolaire et professionelle des filles: des choix de compromise. Une enquête auprés de jeunes femmes issues de familles nombresuses. Revue Française de Pédagogie, 184.

Crowley, K.; Callanan, H. y Allen, E. (2001). Prents explain more often to boys tan to girls during shared scientific thinking. Psychological Science, 12 (3), 258-261.

Dofflon-Novelle, A. (2010). Pourquoi les garçóns n'aiment pas le rise? Pourquoi les petites filles préfèren Barbie a Batman? Perception des codes sexués et construction de l'identité sexué chez les enfants âgès de 3 à 7 ans. Revue Française de Pédagogie.

Draelants, H. y Ballatore, M. (2014). Capital culturel et reproduction Scolaire. Un bilan critique. Revue Française de Pédagogie, 186, 115-142. 
Duru-Bellet, M. (2004). L'école des filles. Quelle formation pour quels rôles sociaux? París: L'Harmattan.

García, J. y Gómez, B. (2012). Diálogos en la cultura de la paridad. Reflexiones sobre feminismo, socialización y poder. Santiago: Universidad.

Granié, M. (2010). Effet de l'adhesión aux stéréotypes de sexe sur les comportements á risque accidentel chez les enfants prèscolaires. En S. Rouyer et al. (2010), 51-62.

LGE (1970). Ley 14/1970 de 4 de agosto, General de Educación y Financiamiento de la Reforma Educativa. BOE, 187, 6 de agosto.

LOE (2006). Ley Orgánica de 2/2006 de 3 de mayo de Educación. BOE, 106 del 4-V.

LOGSE (1990). Ley Orgánica 1/1990 de 3 de octubre de Ordenación General del Sistema Educativo. $B O E, 238,4$ de octubre.

LOMCE (2013). Ley Orgánica 8/2013 de 9 de diciembre para la Mejora de la Calidad Educativa. BOE, 295, 10-XII-2013.

Moreau, M. (2014). Penser l'égalité professionelle entre les hommes et les femmes dans l'enseignement du second degré: une comparaison France-Anglaterre. Revue Française de Pédagogie, 186, 99-113.

Plataforma de Beijing para la Acción: http://www.un.org/womenwatch/daw/beijing/index. html.

Rouger, M.; Croity-Belz, Y. y Prêteur, Y. (2010). Genre et socialization de l'enfant à l'âge adulte: expliquer les differences, penser l'égalité. Toulouse: Érès.

Rouyer, M.; Mieyaa, Y. y Le Blanc, A. (2014). Socialisation de genre et construction des identités sexuées. Revue Française de Pédagogie, 187, 97-137.

Subirats, M. (2014). Avances y retos en las políticas y en las prácticas de los géneros. Educar, especial 30 aniversari, 85-100. 